\title{
Peripherally Inserted Central Venous Catheter Complications in Children Receiving Outpatient Parenteral Antibiotic Therapy (OPAT)
}

\author{
Amanda Kovacich, MD, MPH; ${ }^{1,2}$ Pranita D. Tamma, MD, MHS; ${ }^{3}$ Sonali Advani, MBBS, MPH; ${ }^{4}$ \\ Victor O. Popoola, MBBS, MPH, Sc.M; ${ }^{3}$ Elizabeth Colantuoni, PhD; ${ }^{5}$ Leslie Gosey, RN, MS; ${ }^{6}$ Aaron M. Milstone, MD, $\mathrm{MHS}^{2,3}$
}

овјестіле. To identify the frequency of and risk factors associated with complications necessitating removal of the peripherally inserted central catheters (PICCs) in patients receiving outpatient parenteral antibiotic therapy (OPAT) and to determine the appropriateness of OPAT in children with OPAT-related complications.

Methods. A retrospective cohort of children who had a PICC inserted at the Johns Hopkins Children's Center between January 1, 2003, and December 31, 2013, and were discharged from the hospital on OPAT was assembled.

RESUlts. A total of 1,465 PICCs were used to provide antibiotic therapy for 955 children after hospital discharge. Among these, 117 PICCs (8\%) required removal due to a complication (4.6 of 1,000 catheter days). Children discharged to a long-term care facility were at increased risk of adverse PICC events (incidence risk ratio [IRR], 3.32; 95\% confidence interval [CI], 1.79-6.17). For children receiving OPAT, age of the child (adjusted IRR [aIRR], 0.95; 95\% CI, 0.92-0.98), noncentral PICC tip location (aIRR, 2.82; 95\% CI, 1.66-4.82), and public insurance (aIRR, 1.63; 95\% CI, 1.10-2.40) were associated with adverse PICC events. In addition, 34 patients (32\%) with adverse events may not have required intravenous antibiotics at the time of hospital discharge.

CONCLusions. Of children discharged with PICCs on OPAT during the study period, 8\% developed a complication necessitating PICC removal. Children discharged to a long-term care facility had an increased rate of complication compared with children who were discharged home. With improved education regarding appropriate duration of antibiotic therapy and situations in which early conversion to enteral therapy should be considered, PICC-related complications may have been avoided in $32 \%$ of children.

Infect. Control Hosp. Epidemiol. 2016;37(4):420-424

Peripherally inserted central venous catheters (PICCs) were first introduced in the 1980s, and they have enabled outpatient parenteral antibiotic therapy (OPAT) and reduced lengths of hospital stay. PICCs are occasionally associated with infectious complications, including catheter-associated bloodstream infections, exit-site infections, and tunnel infections, and with noninfectious complications, including venous thrombosis and line breakage. ${ }^{1}$ Data related to the frequency with which PICC complications occur as well as factors that contribute to an increased risk of complications can inform clinicians weighing the relative risks vs benefits associated with PICC placement in patients being discharged on antibiotics. The objectives of this study were to identify the frequency of and risk factors associated with complications necessitating PICC removal in young patients receiving OPAT and to determine the appropriateness of OPAT in patients with subsequent OPAT-related complications.

\section{METHODS}

This study included a cohort of children discharged on intravenous antibiotics from The Johns Hopkins Children's Center with a PICC inserted between January 1, 2003 and December 31, 2013. Patients discharged from the neonatal intensive care unit were excluded. We defined PICCs as peripherally inserted central venous catheters that were placed with the intention of terminating in one of the great vessels near the heart.

The PICC team maintains a database of all children with PICCs and prospectively collects patient and catheter

Affiliations: 1. Department of Medicine, Johns Hopkins Bayview, Baltimore, Maryland; 2. Department of Epidemiology, Johns Hopkins University Bloomberg School of Public Health Baltimore, Maryland; 3. Department of Pediatrics, Division of Pediatric Infectious Diseases, Johns Hopkins University School of Medicine, Baltimore, Maryland; 4. Division of Infectious Diseases, University of Alabama at Birmingham, Birmingham, Alabama; 5. Department of Biostatistics, Johns Hopkins University Bloomberg School of Public Health Baltimore, Maryland; 6. The Johns Hopkins Hospital, Baltimore, Maryland.

PREVIOUS PRESENTATION. The data from this study were presented in part at the 52nd Annual Scientific Meeting of the Infectious Diseases Society of America, Philadelphia, Pennsylvania, October 10, 2014.

Received August 10, 2015; accepted November 24, 2015; electronically published January 12, 2016

(C) 2016 by The Society for Healthcare Epidemiology of America. All rights reserved. 0899-823X/2016/3704-0008. DOI: 10.1017/ice.2015.317 
characteristics, as previously described by Jumani et al. ${ }^{1}$ They document complications necessitating PICC removal both in the hospital and after discharge, by reviewing medical records, and by contacting home care companies or healthcare providers. ${ }^{1}$

Administrative databases were queried for specific demographic information, including insurance status, which is a proxy for socioeconomic status. Patients who developed complications requiring PICC removal were identified. For patients that developed a PICC-associated complication, 2 pediatric infectious diseases physicians reviewed medical records to determine the appropriateness of antibiotic use for those patients with regard to duration and route of therapy based on available evidence and guidelines for conditions including acute and chronic osteoarticular infections, ${ }^{2,3}$ skin and soft-tissue infections, ${ }^{4}$ community-acquired pneumonia, ${ }^{5}$ ventilator-associated tracheitis, ${ }^{6}$ ventilator-associated pneumonia, ${ }^{7}$ urinary-tract infections, ${ }^{8}$ and intra-abdominal infections. ${ }^{9}$ Patient-level characteristics such as age, allergies, and the ability to tolerate medications administered by mouth were considered in the review. Any differences were resolved by discussion until a consensus was reached.

The primary outcome considered in this study was a complication requiring PICC removal, referred to as a PICC complication. PICCs were removed at the discretion of providers and the categorized causes were (1) local infiltration, (2) phlebitis, (3) thrombosis, (4) leakage, (5) occlusion, (6) dislodgement, (7) breakage, and (8) catheter-related bloodstream infection. ${ }^{1}$ To account for severity of illness, patients were categorized as those requiring intensive care (ie, ICU exposure) and those not requiring intensive care (ie, no ICU exposure). The number of days the PICC was in place before hospital discharge was taken into account by the variable "hospital dwell time." Central tip locations were defined as PICCs placed in the arm and terminating in the inferior vena cava, the superior vena cava, or the right atrial junction. Noncentral tip locations were defined as PICCs originating in the arm and extending to any location other than those listed for central tip locations, such as scalp catheters with tips above the level of the clavicle, or lower extremity PICCs with tips below the intravenous (IV) catheter. Children who had a PICC placed previously were identified by the variable "prior PICC." PICC dwell time after discharge (ie, time at risk for a complication) was defined as days from hospital discharge until PICC removal.

\section{Statistical Analysis}

Descriptive analyses were performed to examine patient and catheter characteristics. Poisson regression models were used to estimate the daily risk of PICC complication where the unit of analysis was the catheter. Restricted cubic splines were included to model the nonlinear risk of complication over PICC dwell time after hospital discharge, as were dummy variables for each calendar year to account for temporal trends. Covariates determined a priori to be predictors of complication (ie, age, year, and catheter dwell time) and those with $P<.10$ in bivariate analysis were included in the multivariate analysis. The model produces estimates of the incidence rate ratio of a PICC complication comparing different levels of the covariate. A robust variance estimator was used to account for patient-level clustering and possible violation in the assumed form for the variance based on the Poisson model. This study was approved by the Johns Hopkins University Institutional Review Board with a waiver of informed consent. Data were analyzed using Stata version 13.0 (StataCorp, College Station, TX).

\section{RES ULTS}

During the study period, a total of 1,465 PICCs were used to administer antibiotic therapy in 955 children after hospital discharge. A total of 117 PICCs (8\%) required premature catheter removal due to a complication (4.6 of 1,000 catheter days). Among these, $24(20 \%)$ were infectious complications. Non-infectious complications included occlusion (25\%), dislodgement $(23 \%)$, breakage (13\%), phlebitis (7\%), local infiltration (5\%), leakage (5\%), and thrombosis $(2 \%)$.

Bivariate analyses of potential risk factors for complications necessitating PICC removal are shown in Table 1. The risk of a PICC-related adverse event necessitating catheter removal decreased with age (incidence risk ratio [IRR], 0.93; 95\% confidence interval $[\mathrm{CI}], 0.90-0.96)$, but was higher for children with public insurance (IRR, 1.77; 95\% CI, 1.22-2.57). Noncentral PICC tip locations compared with central tip locations were also associated with an increased risk of complication (IRR, 4.57; 95\% CI, 2.80-7.46). Discharge to a long-term care facility compared with discharge home was associated with an increased risk of PICC complication (IRR, 3.32; 95\% CI, 1.79-6.17). The likelihood of an adverse event necessitating PICC removal was 14.2 of 1,000 catheter days for patients discharged to a long-term care facility (11 of 47 ; $23.4 \%$ ) and 4.3 of 1,000 catheter days for patients discharged home (106 of 1,$418 ; 7.5 \% ; P<.001)$.

In the multivariate analysis including only children who were discharged home with OPAT, the risk of PICC complication decreased by $6 \%$ for every 1-year increase in the age of the child (adjusted IRR [aIRR], 0.94; 95\% CI, 0.91-0.97) (Table 2). The risk of PICC complication decreased by $14 \%$ each year of the study period (aIRR, $0.86 ; 95 \% \mathrm{CI}, 0.80-0.93$ ). Catheter tip location was associated with adverse events necessitating line removal, and noncentral PICCs had a 3-fold higher risk of removal due to complication than central PICCs (aIRR, 3.52; 95\% CI, 2.07-5.91). Public insurance remained associated with an increased risk of PICC complication (aIRR, 1.63 ; 95\% CI, 1.10-2.40).

A total of 11 children (9\%) whose PICCs were removed due to adverse events required PICCs for reasons other than solely for antibiotic administration (eg, parenteral nutrition, frequent transfusions of blood products, etc). For the remaining 106 children who had a PICC at the time of discharge for 
T A B LE 1. Characteristics of Children Discharged with Peripherally Inserted Central Catheters (PICC) on OPAT

\begin{tabular}{|c|c|c|c|c|c|}
\hline Variable & & $\begin{array}{l}\text { PICC Complication } \\
\qquad(\mathrm{N}=117)\end{array}$ & $\begin{array}{l}\text { No Complication } \\
\quad(\mathrm{N}=1,348)\end{array}$ & $\begin{array}{l}\text { IRR (95\% CI) } \\
\text { Unadjusted }\end{array}$ & $P$ Value \\
\hline Sex, No. $(\%)$ & Male & $68(9.58)$ & $642(90.42)$ & Ref & \\
\hline Age per year increase, median (IQR) & & $7(1-13)$ & $11(5-15)$ & $0.93(0.90-0.96)$ & $<.001$ \\
\hline \multirow[t]{2}{*}{ Insurance Sector, No. (\%) } & Private & $67(6.56)$ & $955(93.44)$ & Ref & \\
\hline & Public & $50(11.29)$ & $393(88.71)$ & $1.77(1.22-2.57)$ & .003 \\
\hline Hospital dwell time, median (IQR) & & $1(0-4)$ & $2(1-4)$ & $0.96(0.91-1.01)$ & .140 \\
\hline \multirow{2}{*}{ Tip location, No. (\%) } & Central & $94(6.87)$ & $1,274(93.13)$ & Ref & \\
\hline & Noncentral & $23(23.71)$ & $74(76.29)$ & $4.57(2.80-7.46)$ & $<.001$ \\
\hline \multirow[t]{2}{*}{ Disposition, No. (\%) } & Home & $106(7.48)$ & $1,312(92.52)$ & Ref & \\
\hline & Long-term care facility & $11(23.40)$ & $36(76.60)$ & $3.32(1.79-6.17)$ & $<.001$ \\
\hline
\end{tabular}

NOTE. IRR, incidence rate ratio; PICC, peripherally inserted central venous catheter; ICU, intensive care unit; IQR, interquartile range; Ref, reference.

TABLE 2. Risk Factors for Peripherally Inserted Central Catheter (PICC)-Related Adverse Events in Children Discharged Home on Outpatient Parenteral Antibiotics (OPAT)

\begin{tabular}{llcr}
\hline Variable & & Adjusted $^{\mathrm{a}}$ IRR $(95 \%$ CI $)$ & $P$ Value \\
\hline Sex & Male & Ref & \\
& Female & $0.69(0.46-1.04)$ & .080 \\
Age, y & & $0.94(0.91-0.97)$ & .001 \\
Calendar year & & $0.86(0.80-0.93)$ & $<.001$ \\
Insurance sector & Private & Ref & \\
& Public & $1.63(1.10-2.40)$ & .01 \\
Tip location & Central & Ref & \\
& Noncentral & $3.50(2.07-5.91)$ & $<.001$ \\
Previous PICC & No Prior PICC & Ref & \\
& Prior PICC & $1.39(0.90-2.15)$ & .136 \\
\hline
\end{tabular}

NOTE. IRR, incidence rate ratio; PICC, peripherally inserted central venous catheter; Ref, reference.

${ }^{a}$ Adjusted for catheter dwell time using cubic spline terms and year of PICC insertion.

antibiotic administration only, 27 (25\%) could have transitioned to enteral antibiotic therapy and $7(7 \%)$ could have discontinued antibiotic therapy at the time of hospital discharge. Therefore, 34 patients $(32 \%)$ with an adverse event could have had intravenous antibiotics discontinued and PICC removal prior to hospital discharge.

The scenarios for which antibiotics could have been discontinued or switched to an enteral agent included musculoskeletal infections $(\mathrm{n}=16)$, urinary tract infections $(\mathrm{n}=5)$, skin and soft-tissue infections $(\mathrm{n}=4)$, surgical site infections $(n=4)$, tracheitis $(n=2)$, bacteremia $(n=1)$, intra-abdominal infections $(\mathrm{n}=1)$, and pneumonia $(\mathrm{n}=1)$.

Figure 1 shows the change in complication incidence rate and complication prevalence by year in patients receiving antibiotics through their PICCs after hospital discharge. Both the incidence rate and prevalence of PICC complications after discharge decreased over time (IRR, 0.86; 95\% CI, 0.80-0.93).

\section{I SCUSSION}

In a large cohort of hospitalized children discharged with PICCs on OPAT, $8 \%$ developed a complication necessitating PICC removal. Children discharged to a long-term care facility had increased rates of adverse events requiring catheter removal compared with children discharged home. PICC-related complications may have been avoided in $32 \%$ of children within the group discharged home. These children were discharged with a PICC when they could have either had antibiotics discontinued or switched to enteral antibiotic therapy. This finding emphasizes the importance of improved education focusing on appropriate durations of antibiotic therapy and situations in which early conversion to enteral therapy should be considered.

Few data exist on adverse events associated with OPAT in children. A recent study by Keren et $\mathrm{al}^{2}$ compared clinical and adverse outcomes of post-discharge antibiotic therapy administered orally or via PICCs for children with uncomplicated osteomyelitis. Overall, IV therapy via PICCs was not more effective than oral therapy, but IV therapy had a $15 \%$ adverse event rate requiring an emergency department visit or hospital readmission. Our study only measured adverse events necessitating PICC removal and likely underestimated our true PICC-related complication rate, which likely explains our lower observed number of adverse events compared with Keren et al. ${ }^{2}$ Both studies demonstrate the significant burden of adverse events that should lead to caution in decisions regarding antibiotic therapy at the time of discharge.

Our data suggest that $32 \%$ of PICC-related adverse events may have been prevented with appropriate duration and route of 


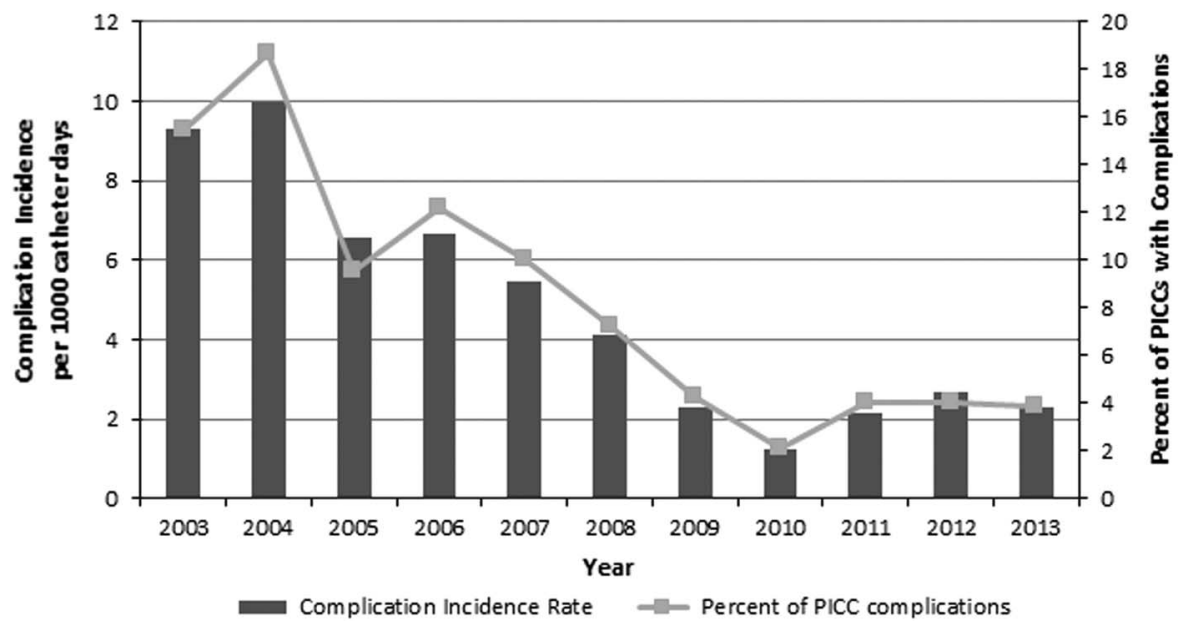

FIGURE 1. Change in complication incidence rate and complication percentage by year in patients receiving antibiotic therapy via peripherally inserted central catheter (PICC) after hospital discharge.

antibiotic therapy. Pediatric-specific data on appropriate durations of antibiotic therapy and conversion to enteral therapy remain limited. However, when including both available pediatric and adult data, guidelines are available for a number of conditions we encountered in our review of cases for which prolonged IV therapy was administered to patients in our cohort, including acute and chronic osteoarticular infections, ${ }^{2,3}$ skin and soft-tissue infections, ${ }^{4}$ community-acquired pneumonia, ${ }^{5}$ ventilatorassociated tracheitis, ${ }^{6}$ ventilator-associated pneumonia, ${ }^{7}$ urinary tract infections, ${ }^{8}$ and intra-abdominal infections. ${ }^{9}$ Mandatory consultations for children being discharged with PICCs for administration of antibiotics could be the tasks of the infectious diseases service or an antimicrobial stewardship team; previous studies have shown a benefit to this approach. ${ }^{10}$ Although it would be difficult to monitor all antimicrobials prescribed upon hospital discharge, focusing on parenteral antibiotics could have a meaningful impact on patient safety.

In some cases, IV therapy is unavoidable. Our study confirms previous findings that younger children and PICCs with non-central tip location incur a greater number of adverse PICC-related events. ${ }^{1}$ The results of the present study provide information relevant to clinicians when providing anticipatory guidance to families at the time PICCs are being considered for their children. Anecdotally, providers often consider sending children at higher risk of complications (eg, younger age or unpredictable social circumstances) to longterm care facilities to complete IV antibiotic therapy. These data suggest that after adjusting for age and other patient characteristics, children discharged to long-term care facilities may not have a reduced risk of adverse PICC events, even after adjusting for age and other patient characteristics (data not shown). Additional studies are needed to carefully weigh the risks and benefits of enteral vs IV therapy in high-risk children, including those discharged to long-term care facilities.

This was a single-center study at a large tertiary, academic medical center, and these results may not be generalizable to other settings. Nevertheless, our study demonstrates unnecessary and avoidable adverse events associated with OPAT and supports the existing literature for consideration of enteral therapy in children with uncomplicated infections. Although patients at particularly high risk for adverse events can be identified and interventions can be implemented, avoidance of OPAT, when possible, is the best strategy for preventing adverse events.

\section{ACKNOWLEDGMENTS}

We thank members of the Johns Hopkins Hospital Pediatric PICC Team for their support of this study.

Financial support. No financial support was provided relevant to this article.

Potential conflicts of interest. AM receives grant support from Sage Products, LLC. All other authors (AK, PT, SA, VP, LG, EC) report no disclosures.

Address correspondence to Aaron M. Milstone, MD, MHS; JHU Departments of Pediatric Infectious Diseases and Epidemiology, 200 North Wolfe St., Rubenstein 3141, Baltimore, MD 21287 (amilsto1@jhmi.edu).

\section{REFERENCES}

1. Jumani K, Advani S, Reich NG, Gosey L, Milstone AM. Risk factors for peripherally inserted central venous catheter complications in children. JAMA Pediatr 2013;167:429-435.

2. Keren R, Shah SS, Srivastava R, et al. Comparative effectiveness of intravenous vs oral antibiotics for postdischarge treatment of acute osteomyelitis in children. JAMA Pediatr 2015;169:120-128.

3. Dubée V, Lenoir T, Leflon-Guibout V, Briere-Bellier C, Guigui P, Fantin B. Three-month antibiotic therapy for early-onset postoperative spinal implant infections. Clin Infect Dis 2012;55: 1481-1487.

4. Hepburn MJ, Dooley DP, Skidmore PJ, Ellis MW, Starnes WF, Hasewinkle WC. Comparison of short-course (5 days) and standard (10 days) treatment for uncomplicated cellulitis. Arch Intern Med 2004;164:1669-1674.

5. Dimopoulos G, Matthaiou DK, Karageorgopoulos DE, Grammatikos AP, Athanassa Z, Falagas ME. Short- versus 
long-course antibacterial therapy for community-acquired pneumonia: a meta-analysis. Drugs 2008;68:1841-1854.

6. Tamma PD, Turnbull AE, Milstone AM, Lehmann CU, Sydnor ER, Cosgrove SE. Ventilator-associated tracheitis in children: does antibiotic duration matter? Clin Infect Dis 2011;52:1324-1331.

7. Chastre J, Wolff M, Fagon JY, et al. Comparison of 8 vs 15 days of antibiotic therapy for ventilator-associated pneumonia in adults: a randomized trial. JAMA 2003;290:2588-2598.

8. Gupta K, Hooton TM, Naber KG, et al. International clinical practice guidelines for the treatment of acute uncomplicated cystitis and pyelonephritis in women: a 2010 update by the Infectious Diseases
Society of America and the European Society for Microbiology and Infectious Diseases. Clin Infect Dis 2011;52:e103-e120.

9. Solomkin JS, Mazuski JE, Bradley JS, et al. Diagnosis and management of complicated intra-abdominal infection in adults and children: guidelines by the Surgical Infection Society and the Infectious Diseases Society of America. Surg Infect (Larchmt) 2010;11:79-109.

10. Shrestha NK, Bhaskaran A, Scalera NM, Schmitt SK, Rehm SJ, Gordon SM. Antimicrobial stewardship at transition of care from hospital to community. Infect Control Hosp Epidemiol 2012;33: 401-404. 